\title{
تعليم اللغة العربية لطلاب اللغة العربية بجامعة بنجكولو الإسلامية الحكومية - إندونيسيا مشكلاته وحلوله
}

Yenni Patriani

Asniti Karni

IAIN Bengkulu

yeyenpatrient72@gmail.com

\begin{abstract}
ملخص
يدور هذا البحث حول "تعليم اللغة العربية لطلاب اللغة العربية بجامعة بنجكولو الإسلامية الحكومية -

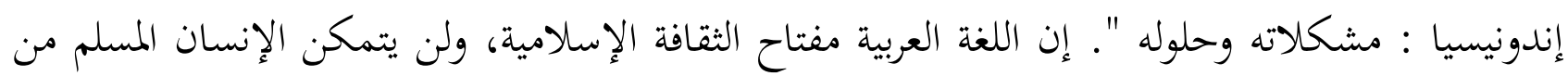

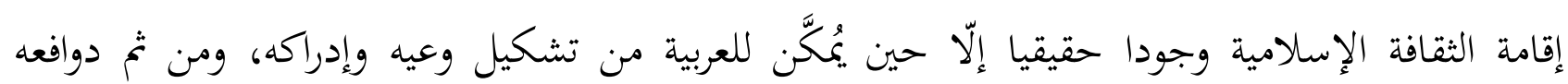
وسلوكياته. وإن القرآن الكريم والحديث الشريف هما السبب في نشأة الدراسات العربية بغروعها المختلفة بإندونيسيا وخاصة بجامعة بنجكولو الإسلامية الحكومية. وكانت هذه الدراسات أساسا لخدمة الإسلام ولفهم القرآن الكريم والحديث الشريف. على الرغم من طول عهد الإندونيسيين باللغة العربية التي اقترنت

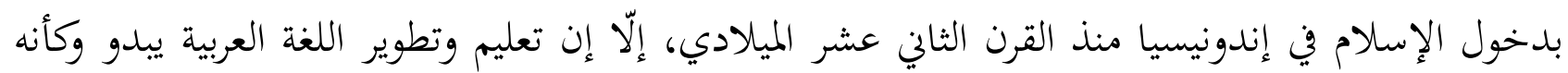

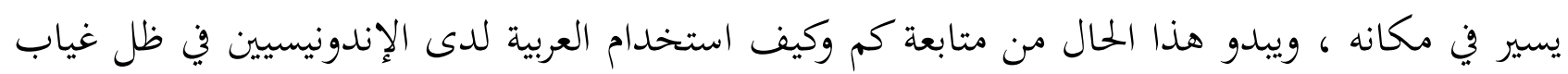
شبه تام للأنشطة البحثية والإبداعية باللغة العربية. وفي هذا البحث أجريت دراسات كثيرة تناولت تعليم اللغة العربية لطلاب جامعة بنجكولو الإسلامية الحكومية وتحاديات تعليم اللغة العببة في هذه الجامعة وحلوله. ويتبع هذا البحث المنهج الوصفي باعتباره المنهج الملائم لطبيعة البحث. لئه
\end{abstract}

الكلمة المفتاحية: تعليم اللغة العربية; طلاب جامعة بنجكولو الإسلامية الحكومية; المشكلات والحلول

\begin{abstract}
This paper is about "Teaching Arabic to Arabic Language Students at Bengkulu Islamic State University - Indonesia: Problems and Solutions . The Arabic language is the key to Islamic culture, and the Muslim will not be able to establish Islamic culture in real existence except when he give the Arabic consciousness to motive and behavior. The Holy Quran and the Hadith are the reason behind the emergence of Arab studies in various branches in Indonesia, especially the Bengkulu Islamic State University. These studies were mainly to serve Islam and to understand the Holy Quran and the Hadith. Despite the length of the Indonesian era in Arabic, which has been associated with the entry of Islam in Indonesia since the twelfth century AD, the teaching and development of
\end{abstract}


the Arabic language seems to be in place. This seems to follow how and how Arabic is used by Indonesians in the near absence of research activities And creative in Arabic. In this research, many studies have been conducted on the teaching of Arabic language for students of Bengkulu Islamic State University and the unity of Arabic language teaching at this university and its solutions. This research follows the descriptive approach as the appropriate method for the nature of research.

Keywords: Teaching Arabic; Students of Bengkulu Islamic State University; Problems and solutions

مقدمة

أ.نبذة عن جامعة بنجكولو الإسلامية الحكومية

تقع جامعة بنجكولو الإسلامية الحكومية في مدينة بنجكولو في جزيرة سومطرة، إندونيسيا. بداية

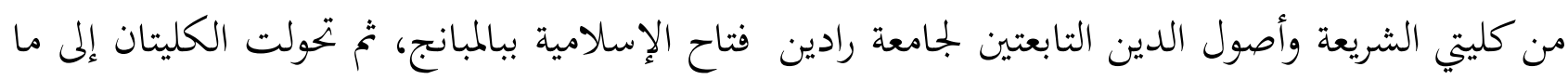
يسمى بالمعهد العالي الحكومي للعلوم الإسلامية ببنجكولو والمعهد العالي الحكومي للعلوم الإسلامية

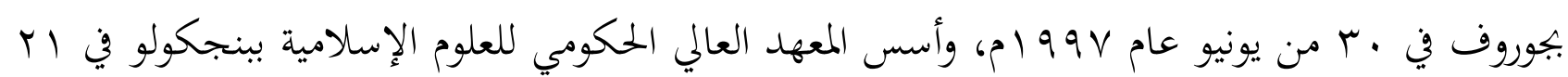

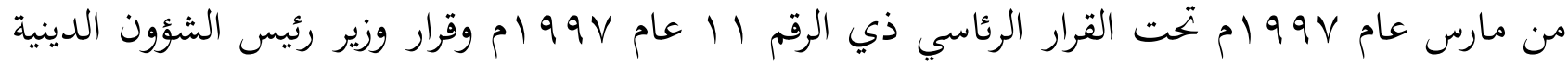

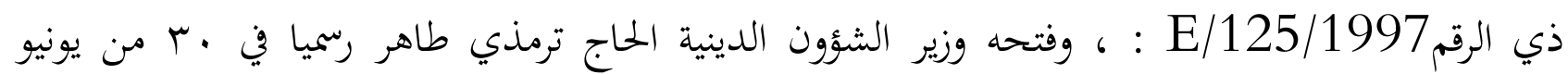

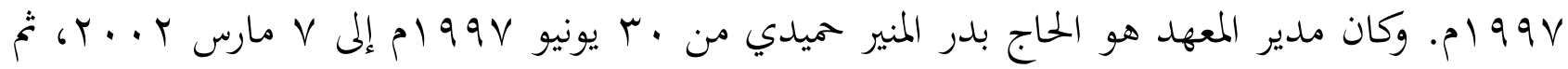

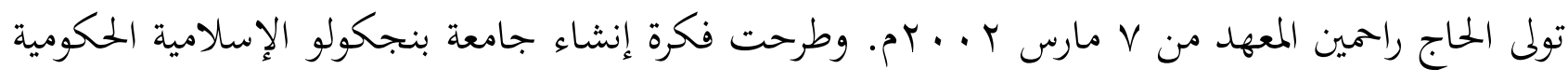

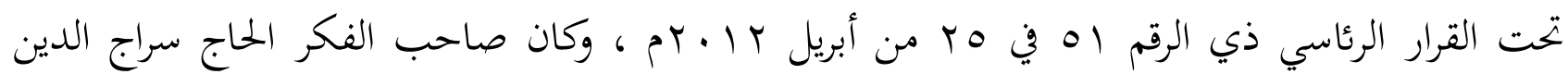

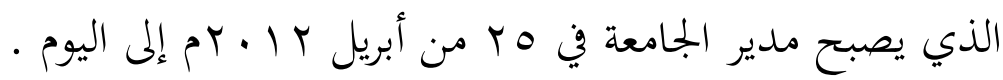

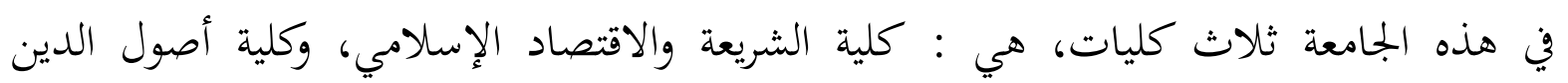
والدعوة والآداب، وكلية التربية والتعليم. أما اللغة العربية فهي من كلية التعليم.. وعدد الطلاب في هذه القسم .0 10 10 مالبا. ب.منهج تعليم اللغة العربية بجامعة بنجكولو الإسلامية الحكومية وأهدافه ا ـ. منهج تعليم اللغة العربية

ينقسم تعليم اللغة العربية في هذه الجامعة إلى ثلاثة أقسام: المنهج الوطني، والمنهج الجامعي، ومنهج الكلية. المنهج الوطني هو الذي وضعته وزارة الشؤون الدينية ويجب مراعاته في بناء مناهج تعليم اللغة العربية

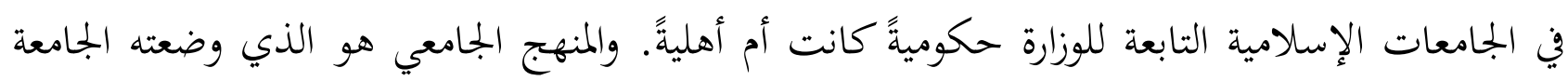
خاصة بها ويجب مراعاته في تعليم اللغة العربية في الكليات التابعة لها. ويختلف هذا المنهج من جامعة إلى

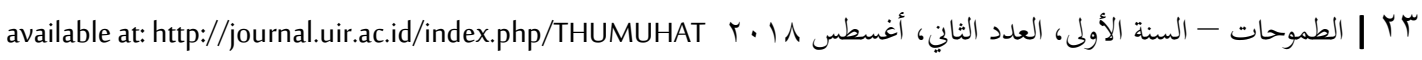


أخرى إذ إنه عبارة عن ابتحه تسير عليه كل جامعة في تعليم اللغة العربية. أما منهج الكلية فهو الذي وضعته

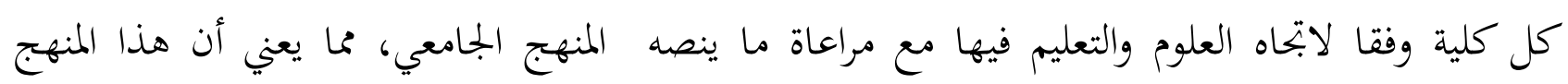
يختلف من كلية إلى أخرى. r. أهداف تعليم اللغة العربية

إن الأهداف العامة من تعليم اللغة العربية في هذه الجامعة هي الأهداف التأهيلية والمهنية. وتسعي

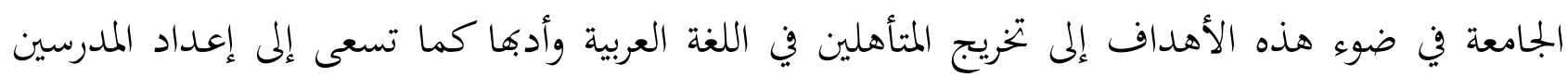
المهنيين في حقل اللغة العربية. ولقد اكتشفت الباحثة خلال مسحها الميداني في هذه الجامعة أن هذه الأهداف في الواقع لم تتحقق. وذلك لعدة أسباب يتلخص أهمها كما يلي: أ. ألمنهج التعليمي

المنهج التعليمي الذي وضعته الجامعة في تعليم اللغة العربية يتركز من حيث الأهداف على الجوانب اللغوية البحتة مثل الإلمام بالقواعد والترجمة والمفردات نتيجة تقيد تعليم اللغة العربية بالأهداف الدينية. إن الإلمام بهذه الجوانب اللغوية لا يمثل ما يحتاج إليه الطلاب ليكونوا متأهلين في بجال اللغة العربية.

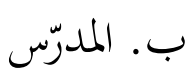

وإن المشكلات التي ترجع إلى تعليم اللغة العربية وتتعلق باللغة والمدرّسين من أسباهما ما يلي : هتخدام بعض المدرسين اللغة الإندونيسية في تعليمهم. ازدواجية اللغة المتمثلة،العربية يُدرسبها والإندونيسية يمارسوفا خارج البيئة الدراسية. وجود مشكلات نطقية لدى بعض المعلمين. عدم العمل بروح الجماعة التي تساعدكثيرا في حل المشكلات اللغوية لدى الطلاب. عدم كفاءة بعض المدرسين واستخدامهم طرقا تقليدية في تعليم العربية كلغة تركية ثانية. توحيد طريقة التعليم بين الطلاب المتخرجين من المدارس العامة والمتخرجين من المعاهد أو المدارس الإسلامية. شرح المدرسين باللغة الإندونيسية أو اللهجات التي لا تتفق مع الفصحى. 
وإن معلمي اللغة العربية في هذه الجامعة كان معظمهم لا يتمتعون بالخبرات اللغوية الكافية

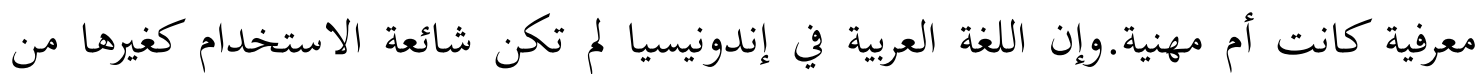
اللغات الأجنبية. إنما تتعلمها وتستخدمها فئات معينة من الإندونيسيين في بيئات محدودة. إن معظم مدرسي اللغة العربية في الجامعة الإسلامية الحكومية هم أهل البلد وليسوا اللغة. قليل من المدرسين من سبق لمم أن يتعاملوا مع العرب لمدة طويلة، ولذا عندما يجد هؤلاء المدرسون صعوبة في التعبير فيقومون بمعالجات فردية دون اللجوء إلى الناطقين الأصليين. ج. المواد التعليمية. أما من حيث المادة فتعليم اللغة العربية في هذه الجامعة يلجأ إلى الكتب النحوية مثل والنحو الواضح، والنحو الوافي، وجامع الدروس العربية وغيرها من الكتب النحوية. والتعليم باستخدام هذه

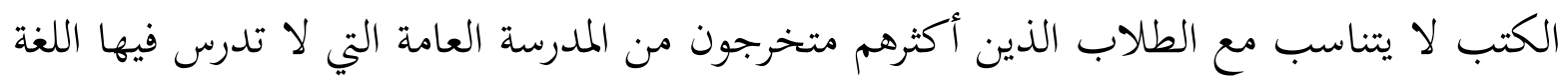

$$
\text { العربية. }
$$$$
\text { د. الطلاب }
$$

المشكلات التي تواجه الطلاب في تعليم اللغة العربية لغير الناطقين بها ترجع إلى الطلاب

$$
\text { أنفسهم نتيجة للأسباب التالية: }
$$

عدم رغبة الطلاب في تعلم اللغة العربية ويظهر هذا من عدم جهودهم.

عجز بعض الطلاب عن استيعاب الجديد خاصة إذا تزايد الكم المقدم.

بعض الطلاب متخرجون من المدرسة العامة التي لا تدرس فيها اللغة العربية.

عدم حفظ بعض الطلاب القرآن الكريم.

بعض الطلاب غير قادرين على نطق الأصوات لوجودمشكلات عندم: لعنده.

مرور بعض الطلاب بخخبرة ضعيفة بتعلم العربية مما أثر في نفوسهم عندإعادةتعلمهم هذه الهيته

اللغة.

وإن المشكلات التي تعود إلى اللغات الأم عند الطلاب تتلخص أسباهمافيمايلي:

أن العربية لغة إعراب، والإعراب مشكلة عند الطلاب الناطقين ببعض اللغات. صعوبة نطق بعض الأفعال لدى الطلاب لاختلاف عين الفعل.

لايستطيع الطالب التفريق بين الأصوات المرققة التي لما أصوات مطبقة من نفس نوعها. 
افتقاد المنهج الواضح الذي يهكم بعض الظواهر اللغوية مثل جمع التكسيرمثلا. وجود تشابه بين الحروف سواء في نطقها أوفي كتابتها. كثرة المترادفات في العربية تؤدي إلى الاضطراب التعليمي لدى الطلاب. وجودصعوبة في فهم القضايا البلاغية والتشابه بعضها البعض. وجود أصوات تنطق ولاتكتب وحروف تكتب ولاتنطق. عدم ضبط الحروف العربية بالشكل في بعض الموادالمطبوعة. توحيد الكتاب المدرسي لدى المبتدئين بالرغم من اختلاف مستوى الطلاب المبتدئين، فمنهم من عنده خلفية لغوية عربية ومنهم من يفقد هذه الخلفية بل تنعدم عندهم أي معرفة باللغة العربية وأنظمتها. هـ. طريقة التعليم

الطريقة الشائعة الاستخدام في تعليم اللغة العربية في هذه هي طريقة القواعد والترجمة تلك الطريقة التي تعد أقدم طرائق تعليم اللغات الأجنبية والتي لم تعد تتناسب مع الابحاهات الحديثة في تعليم اللغات الأجنبية. وهذه الطريقة قد بتعل الطلاب يسأمون.

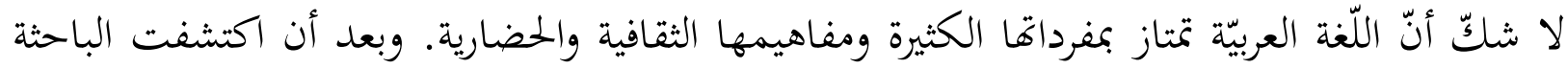
المشكلات في تعليم اللغة العربية، رغبت الباحثة في تقديم الحلول التي يمكن أن يستفيد منها المعلّمون والمتعلّمون في تعليم اللغة العربيّة، وتلك الحلول هي : ا. ينبغي لمعلّمي اللغة العربيّة، خاصّة في جامعة بنجكولو الإسلامية الحكومية أن يقوموا بتعليم اللغة العربية بتعليم محتواها الثقافي. r. استخدام كتب اللغة العربية التي ألّها أهل اللغة لتعليم اللغة العربية في المدارس والمعاهد الإسلامية. r. إعداد دورات تدريبّة لمعلّمي اللغة العربيّة في مجال استعمال اللغة العربية الأصلية. 


\section{المراجع}

دوجلاس براون، أسس تعلم اللغة وتعليمها، ترجمة عبده الراجحي وعلى أحمد شعبان، دار النحضة

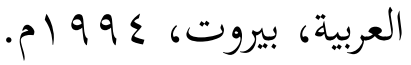

رشدي أحمد طعيمة، تعليم اللغة العربية لغير الناطقين بها: مناهجه وأساليبه، منشورات المنظمة

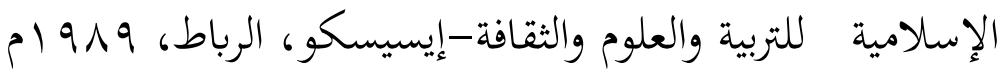

عبد المجيد سيد أحمد منصور، علم اللغة النفسي، عمادة شؤون المكتبات-جامعة الملك سعود، الرياض، .p $191 \mathrm{r}$ على تُمَّم القاسمي، ابحاهات حديثة في تعليم العربية للناطقين باللغات الأخرى، عمادة شؤون المكتبات

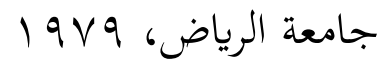

مُعَّمَ على الخولي، أساليب تعليم اللغة العربية، طب، الياض، 919 ام. مُعجّ على الخولي، الحياة مع لغتين، دار الفلاح للنشر والتوزيع، الأردن، ب + . بم. محمود كامل الناقة ورشدي أحمد طعيمة، الكتاب الأساسي لتعليم اللغة العربية للناطقين بلغات أخرى: إعداده-تحليله-تقويمه، جامعة أم القرى، مكة المكرمة، بمدو ام. نبيه إبراهيم إسماعيل، الأسس النفسية لتعليم اللغة العربية للناطقين بغيرها، مكتبة الأنجلو المصرية 